vulsions. Santonin and calomel were given; but the fits recurred, and he died in thirty-three hours, after having upwards of thirty fits. At the necropsy all the viscera were carefully examined, but nothing was found to explain the convulsions except two round worms in the small intestine.

Further exact records of the condition of the viscera in fatal cases of convulsions associated with worms are much needed. It is possible that violent and prolonged convulsions may in some cases produce such conditions as cerebral effusion or volvulus. Be this as it may, we cannot ignore the great importance of a thorough knowledge of the pathology of ascarides in the tropics.

$=i$

\section{Ankylostoma Duodenale.}

Though the valuable researches of Lutz have cleared up many points in the life history of this worm, we have still much to learn as to the pathology of the symptoms which it induces. For example, is the intense anæmia, which is the chief characteristic of ankylostomiasis, the result of a simple drain of blood from the intestine by the worms, or is it, as William Hunter contends, the result of excessive blood destruction in the portal circulation effected by the action of certain poisonous agents absorbed from the intestinal tract through breaches of surface made by the worms? Analysis in four cases of ankylostomiasis, which $\mathrm{Mr}$. Casmody and $\mathrm{Mr}$. Tate have been kind enough to make for me, have shown far less iron in the liver than is usually found in cases of pernicious anæmia. So far, then, the theory of a simple drain of blood is supported, but I am waiting for more cases before summarising my conclusions. Another very important question is the relation of dirt eating to the presence of the parasite. Lutz regards geophagy as a symptom of ankylostomiasis, but it must also be admitted that fresh parasites may be introduced into the intestine with the earth. Again, severe and fatal cases of earth-eating are sometimes met with in which no ankylostomata are present. On this point many more observations are needed.

\section{AN ADDRESS}

ON THE

\section{TREATMENT OF CANCER OF THE BREAST.}

Delivered before the Harveian Society of London.

By W. WATSON CHEYNE, M.B., F.R.C.S.,

Professor of Surgery in King's College: and Surgeon to King's College Hospital.

I THIN that this subject cannot be too often brought before the notice of the medical public. First, because the disease is common, at any rate in certain regions, and seems to be becoming more so. Secondly, because the necessary operation is looked on as a very simple one, and is now done by many practitioners, even by those who do not undertake the more serious operations of surgery. Following the description found in most textbooks of surgery, all that is thought necessary is to enclose the nipple and areola in an elliptical incision extending from the inner to the outer side of the prominent part of the breast, dissect the skin off the rest of the breast till one thinks that one has got beyond it, then deepen the incision to the muscle all around, and, seizing the breast, pull it off the muscle quite regardless as to whether the pectoral fascia comes with it or not. Further it is often taught that if no glands are felt in the axilla it need not be opened, or if glands are felt the finger should be introduced and the enlarged glands pulled out. I do not of course mean to imply that this is the common practice with surgeons at the present time, but it was so till quite recently, and has been and is so taught in some textbooks. This method is wrong in every particular, and this leads me to the third reason why it seemed desirable to bring the subject forward, namely, that researches have been made within quite recent years, notably by Heidenhain, Stiles, Raymond Johnson, and others, which have given us much more precise knowledge as to the amount of tissue which ought to be removed in these operations, and it is well that these researches should be kept before the medical public and not be lost sight of.

What is the object of operation in cases of cancer of the breast? Some still look on cancer as a constitutional disease, and therefore only operate with the view of taking away the local manifestation and prolonging life, and they reckon that by operation twelve to eighteen months are added to the patient's life. They expect recurrence as a matter of course, and therefore do not pay very great attention to the exact details of the operation. Others look on the disease as essentially local, and therefore operate in the hope of ridding the patient of it entirely-in fact, of producing a cure. There are, I think, very few at the present day who do not adopt the view that the disease is essentially local in the first instance, and that distant parts become attacked as the result of direct infection from the primary growth. We know from pathology that malignant epithelial growths only begin in epithelial structures, and cannot commence in lymphatic glands, cellular tissue, etc.; and, therefore, recurrences taking place in these tissues after operations on the breast cannot mean a fresh manifestation of a constitutional disease, but must imply the growth of portions which have been left behind at the time of the operation. And, further, we know clinically that these local recurrences usually occur within a short period after the operation - six to eighteen months as a rule-and that if a patient remains free for a longer period, there is a very considerable probability that she will remain free permanently. As a matter of fac $i$ the relation of the constitution to cancer is, I believe, practically the same as its relation to tuberculosisnamely, that there are certain persons in whom the disease will spread more rapidly and readily than in others, but that in all there must be some point of commencement, where the disease is in the first instance purely local. The chief argument in favour of the constitutional nature of the affection is the occurrence of cases where the second breast becomes the seat of disease after the commencement of the disease in, or the removal of, the first. Recent researches have, how 'ver, shown that in some persons, especially those with extensive mammæ, the lymphatic systems of the two breasts intercommunicate, and hence a direct spread of the infective material from one to the other is quite possible; and, as adding support to this view, is the fact that in these cases the primary growth is generally situated towards the sternal side of the breast first affected.

Further evidence of the local origin of cancer is furnished by the results of operation. Even with the older methods of operating a certain number of cases taken in hand at an early period of the disease remained free from recurrence, and more recently the number has increased in correspondence with the greater completeness of the operation, so that a very general estimate of 10 to 15 per cent. of permanent recoveries is given. Since the publication of Heidenhain's researches, however, surgeons who have acted on his facts produce better results, and in the recent French treatise on Surgery statistics are given where in one case as many as 22.5 per cent. were free from recurrence after three years. It must be noted, however, that this does not show the full recent improvement, because some of these were operated on several years ago, and I believe better results ought really to be obtained. Success must essentially depend on operation at the earliest possible period and on the thoroughness with which this operation is carried out.

\section{Early Operation.}

Early recognition of the disease and early operation cannot be too strongly insisted upon. Unfortunately, cases are too often watched and treated with various useless remedies till the infection has spread to such an extent that the possibility of getting beyond it by operation has become very doubtful. This is generally, no doubt, the fault of the patient, or her misfortune, in that she has not happened to notice the growth, and it cannot be a matter of surprise that, especially with the ordinary limited operation, recurrence should so frequently take place. Where one is certain that the disease is cancer and that the case is suitable for operation, delay is naturally objectless and unjustifiable, and of course it is only cases in which the diagnosis is doubtful which are watched. 
The chief difficulty in diagnosis at an early stage is from chronic lobular mastitis, either simple or tuberculous; it is not often that the other tumours cause much difficulty. In the case of mastitis, the swelling is not usually so hard ; it is distinctly tender; its enlargement is sometimes more rapid than cancer, especially when tuberculous; the enlargement of the axillary glands occurs earlier and is often greater and the glands are tender, and it is often a direct sequela of a pregnancy or a lactation. In the case of cancer the skin tends to wrinkle over the region of the tumour, when the breast is pushed to one or other side in a way which does not occur in mastitis till the inflammation has progressed considerably.

I do not propose here to go into the differential diagnosis of scirrhus of the breast. The question I wish to ask is, Why watch a lump in the breast if one has any suspicion that it may be cancerous? If it should turn out to be a cancer, then precious time has been lost, and it is of essential importance, from the point of view of permanent cure, that a decision should be come to without delay. What is no doubt in the practitioner's mind is that it would be a great pity to excise a breast and then find that after all it was only inflammatory; but the question of excision does not really come into play; an exploratory incision will usually settle the question. Cut into the swelling and remove a slice, and if it is scirrhus it can generally be recognised at once with the naked eye; if there still remains a doubt, the part removed can be submitted to microscopical examination. As a matter of fact, I should advise, if the lump is small, to remove it altogether rather than a slice of it; if it is a cancer there is none of the juice left in the wound, and if it is inflammatory the disease is taken away. Is there any objection to the exploratory incision? None whatever. If it turns out to be inflammatory a free incision is the best method of treatment, while if it be cancer the diagnosis is made at once. If done antiseptically the small wound does not give rise to any trouble, and the scar left need not be large.

\section{Method of Removal.}

If now we have to do with a cancer of the breast, ought we to be content with the incision I referred to at the beginning, and either leave the glands alone, or only pick out those which are enlarged? The answer is No, because the study of the nature and mode of extension of the disease shows that by such a method of operating we must, unless in cases which are early and very limited, leave material which has almost certainly become infected already. Cancer of the breast begins as an epithelial proliferation in the acini or ducts of the breast, but the growing epithelium very soon bursts through the wall of the tube and gets into the lymph spaces and lymphatic vessels, and the history of its further spread is intimately bound up with the anatomy of these vessels, for having once got into the lymphatic system portions become broken off, and are carried on with the lymph stream forming emboli either in the course of the vessels, or in the nearest lymphatic glands. Now, according to Sappey, the lymphatic vessels of the breast commence as a fine plexus around the acini, from which they run along the ducts, forming a plexus around them, to the nipple. From thence they pass into the fat, and a large plexus is formed under the areola which is joined by lymphatics from the skin, and is called by Sappey the subareolar plexus. From thence three or four large lymphatic vessels pass to the axillary glands. Since Sappey published his researches, it has been found that this is by no means the only route which the lymph takes from the breast. In cases where the cancer is situated in outlying lobules, or near the deeper surface, it gets into the lymphatic system of the pectoral fascia, which also goes to the axillary glands. Further, from the sternal end lymphatics apparently run to the sternum and sometimes to the other breast, and, at any rate if the growth has become adherent at the inner end they also run, I believe, to the mediastinal glands. Again, lymphatics run from the breast to the skin in the bands of fascia which connect the two-the suspensory ligaments of the breast. It must also be borne in mind that the pectoral fascia is intimately connected with the surface of the pectoral muscle, and can hardly be removed thoroughly without taking away also a thin layer of the surface of the muscle. Now, as the infective material may have passed along the lymph- atics at an early period of the disease, it is necessary, in order to give the best chance against recurrence, to remove as far as possible the whole lymphatic area up to and including the nearest lymphatic glands, that is, the axillary glands, and that whether the disease in these parts is large enough to. be felt or not. To remove the growth or even the breast. alone may be sufficient in a few cases, but we know from clinical experience that it is not so as a rule. To remove the breast and the enlarged lymphatic glands is also not sufficient, because the vessels leading to these glands may be, and are very often, also infected. To remove the enlarged lymphatic glands and leave the fat and other glands which are not enlarged is also not sufficient, because in the fat lymphatic vessels run which may be infected, and once one gland is attacked the disease quickly extends to the others, which may be diseased although not yet enlarged. Hence, as I have said, the whole lymphatic area should be excised in alp cases. It is not the visible disease which is the difficulty; we have here, as in aseptic treatment, to contend against the invisible, and, just as in aseptic treatment, our results will be good in accordance with the care with which we act against. the invisible.

Therefore in excising the breast for cancer, we must, in the first instance, remove the whole breast, and in this connection we must bear in mind the facts which have been admi-rably demonstrated by Mr. Harold Stiles that the breast is a much more extensive organ than has been supposed, and that if only the prominent breast is removed, a ring of lobules will be left all around which may contain infectivematerial and subsequently give rise to disease. In order to. remove the whole breast, which extends over the greater part, of the front of the thorax, it is necessary, or at any rate advisable, in all cases to remove the skin coextensive with the prominent part of the breast. No definite skin incisions can be described, because skin must never be dissected from over a tumour on account of the fibrous bands running to the skin to which I have already referred; where tumours lietowards the edge of the breast, special incisions must be made, not only to take away the skin over them, but for a. considerable area around them. Further, the pectoral fascia must be removed coextensive with the breast and this is readily done by taking a thin layer of the muscles along with it. Then, lastly, the fat and fascia leading from the breast to theaxilla, and the whole of the fat and glands in the axilla, should be dissected, not pulled, away in all cases. I do not. propose here to go into the question of what should be donein advanced cases, but I may say that when the tumour is at. the inner end of the breast, the incision should be carried well over the sternum, and the fat and fascia extensively removed in that region. In the great majority of my cases, $\mathbf{I}$ have been able to stitch up these wounds completely afterundermining the skin, and using button and wire sutures; if any small area is left, it can be readily covered by a few skin grafts.

My remarks are not founded on mere theory, they are based on well-ascertained pathological facts, and the results which have been obtained fully bear them out. I have not myself a large number of cases to bring forward, but I shall mention the results of the first 20 cases on which $I$ have operated in this thorough manner. I take the first 20 because the remainder have been operated on too recently to be of value. I began operating in this thorough manner in the beginning of 1890 , and the first 20 cases in which I did the first operation (I am not referring to operations on cases of recurrence operated on in the first instance by other surgeons) bring. me down to near the end of 1892 . Thus, in all over a year and in some from three to four years have elapsed since the. operation. Of these 20 cases, 14 remain well, and 6 have recurred. But it will be better to consider only the cases: operated on in 1890 and 1891, that is to say, cases in which more than two years has elapsed since the operation. Of these there are 15 , of which 10 remain well, and 5 have recurred and the patients have died of the recurrence. This: would give a percentage of over 66 per cent. of cases remaining well for over two years. I may mention the length of time which has elapsed in each of these 10 cases : 4 years;: 3 yrs. 8 mos.; 3 yrs. 3 mos.; 2 yrs. 11 mos.; 2 yrs. 10 mos.: 2 yrs. 3 mos. (2 cases); 2 yrs. 2 mos.; 2 yrs. 1 mo. (2 
cases). In the cases which recur.ed, the disease reappeared in a year or less, except in one case where neariy eighteen months elapsed before internal cancer was diagnosed. Now, I do not for one moment mean to say that all of the 10 cases still free from disease after two years or longer will permanently remain so, nor that 66 per cent. is. anything like the proportion of permanent recoveries that can be hoped for, but between 66 per cent. and 10 per cent. there is a great interval, and several of these cases might recur and still leave a better percentage than that got by the older methods of operating; in fact, only one need survive to give a result very much like that obtained by the old method, and I do not think I am too sanguine in thinking that more than one will remain free from disease. We know that where local recurrence takes place it usually does so within the first year, and Volkmann has formulated the following law, founded on his experience: "When, after an operation, a whole year has elapsed without recurrence one may hope for a permanent cure; after two years this cure is probable; at the end of three years it becomes almost certain." Of these 15 cases, 5 were operated on more than three years ago, and of these 3 , or 60 per cent. remain well, and come under the third part of Volkmann's law which says that cure is almost certain. I would further remark, with regard to these cases, that none of them were early cases; in all the axillary glands could be felt enlarged before the operation and were found to be diseased at the time. I may, however, remark that in some of the cases the disease had been progressing slowly. As local recurrences are always due to disease left behind it is only what we should expect that the freer the operation the more likely one is to have removed all the disease. Of course, if the infection has passed beyond the axillary glands no extent of operation will remove the disease, and that, of course, brings us back again to the advisability of early operation. It seems to me that the pathological and clinical facts being as I have stated, even those who adopt the constitutional view, and who only operate with a view of prolonging life, must also adopt the more thorough operation.

The objections which are mentioned against the complete operation are mortality and interference with the freedom of movement of the arm.. As regards the question of mortality, if the operation is performed aseptically the"mortality will be very small, and may be neglected. In all my cases of excision of the breast, which, including extensive recurrences and some not so complete as above described, number about 50 , I have only lost one case. This was a lady operated on a year ago for very extensive and immediate recurrence after excision on the old lines by another surgeon. I had not only to remove the greater part of the skin from the side of the chest, but also the pectorals, all the axillary fat and glands, and some glands from the neck; in fact, had I had any idea before I began that the disease was so extensive, I would not have operated. She developed pneumonia on the side operated on immediately after the operation, possibly as the result of the exposure in the cold weather, and died in a week. Allowing for accidents such as this, I do not believe that the mortality after the complete operation ought to be more than 2 or 3 per cent. if proper antiseptic precautions are taken. As regards the movement of the arm, there is no doubt a certain amount of restriction of movement after thoroughly clearing out the axilla, but all my patients can at least do their back hair afterwards, and $I$ think if a patient understands that this operation gives her a much better chance of permanent recovery, and that she will be able to do her back hair, very few will think that a little restriction of movement is worth considering.

Cases Not Suttable for Operation.

With our present knowledge we can no doubt operate on more extensive cases than formerly with a fair prospect of success. At the same time I doubt if those very extensive cases in which the pectorals have to be removed or the clavicle divided are really suitable, and I should not myself advise operation in cases in which enlarged glands could be felt above the clavicle, in which several nodules can be felt in the muscles, in which there are numerous scattered nodules in the skin, or in which there is œedema of the arm, with pain down the arm, implying adhesion to the struc- tures in the axilla. As to atrophic cancer, I sec no reason why it also should not be removed unless the patient is very old and feeble.

The following is an abstract of the ten cases previously reerred to, which remain well after more than two years. I may say that all of these cases were undoubtedly scirrhus as confirmed by microscopical examination.

1. Aged 52, operated on in January, 1890. Nipple had been retracted for about two years; tumour size of hen's egg, close to nipple, involving the skin : numerous small, hard glands to be felt.

2. Aged 38, operation, May, 1890 . Tumour noticed two months, size of small orange, only slight puckering of skin, breast moves freely on muscle, a number of small, hard glands.

3. Aged 50, operation, October, 1890. Eczema of nipple for two years, lump noticed in breast for six months, close to nipple, several enlarged glands.

4. Aged 47, operation, February, 1891. Swelling noticed for two years, size of hazel nut, involving skin, glandular enlargement slight.

5. Aged 52, operation, March, 1891. Pain and a little swelling for nearly two years; tumour size of walnut, close to nipple, skin adherent and ulcerated, only slight glandular enlargement.

6. Aged 44, operation, October, 1891: Noticed lump and retraction of nipple for six months. Tumour size of orange, several glands, one of them large.

7. Aged 63, operation, Otober, 1891. Retraction of nipple noticed for eighteen months; small but diffuse scirrhus; a number of small, hard glands.

8. Aged 56, operation, November, 1891. Nodule first noticed three years ago, growing rapidly for last three months, size of hen's egg, glands considerably enlarged.

9. Aged 49, operation. December, 1891. Noticed lump for ten months, size of pigeon's egg ; skin puckers markedly, glands small but hard.

10. Aged 62, operation, December, 1891. Lump noticed for three years, skin adherent but not ulcerated; numerous small, hard glands.

\section{THE TREATMENT OF OVARIAN AND EXTRA- UTERINE CYSTS COMMUNICATING WITH THE RECTUM. ${ }^{1}$}

BY HARRISON CRIPPS, F.R.C.S.,

Operator for Abdominal Sections in the Women's Ward, St. Bartholomew's Hospital.

WITH an experience of over 100 cases of ovariotomy, the four recorded below are the only instances of a fistula between the cyst and the rectum that I have met with, and it would be of interest to learn from more experienced operators whether this complication is as common as it has unfortunately been in my practice.

CASE I.-M. A., a nurse, aged 25, was invalided from the hospital in June, 1892, on account of persistent pyrexia (night temperature ranging from $101^{\circ}$ to $\left.102^{\circ}\right)$. She complained of no pain, and the pelvis was not examined. Two months later she had considerable pain in the left iliac fossa, and soon afterwards noticed an enlargement. The pain increased, and became very severe during the first week in October. A sudden discharge of over a pint of pus then took place per rectum. Two days later, on examination, the abdomen was found distended and very tender over the left iliac fossa. No distinct tumour could be felt from above. Per vaginam the cervix was pushed over to the left. In Douglas's pouch a nodulated swelling could be felt, but not very clearly defined. Per rectum, at a distance of 4 inches from the anus and just within reach of the finger tip, a small opening with ragged edges could be felt in the front wall. This obviously led into the swelling mentioned. For some days the patient was much relieved, but after that time the discharge days the patient was much relieved, but after that time the discharge
was less, and the cavity appeared to be only partly drained. Pyrexia rewas less, and the cavity appeared to be only partly drained. Pyrexia reappeared, and the swelling could be more distinctly telt per vaginam. During Dr. Champneys's temporary absence the patient came under the care of Dr. Griffith. After consultation, and on grounds to be presently considered, it was determined to drain the cyst or abscess cavity by puncture through the vagina. This was accordingy done, and a glass tube inserted. The result was satisfactory, the discharge diminishing. and the pyrexia disappearing. The patient left the hospital at the end of November, with no discharge from the rectum and scarcely any from
the vagina. She was readmitted two months later. She had had no pain since leaving the hospital, the temperature being normal. She had passed no pus by the rectum, but had always had a slight discharge by the vagina. Her general health had greatly improved. On examination an elastic swelling could be felt on the left side, reaching nearly to the
navel. No opening could be felt by the rectum, but there was a hole admitting a probe in the posterior quarter of the vagina

Assisted by Dr. Champneys I performed abdominal section. On the abdomen being opened a thin-walled cyst presented itself, spreading up to the left side and behind the posterior layer of the broad ligament. The cyst was tapped, and two pints of clear fluid were let out. At the bottom of the cyst, after evacuation, another cyst with very thick walls could be felt firmly embedded in the pelvis, between the uterus and rectum. This second cyst was tapped through the broad ligament cyst which had already been opened. A considerable quantity of gas and which had already been opened. A considerable quantity of gas and
from 3 to 4 ounces of greenish pus escaped. The pus being thooroughly from 3 to 4 ounces of greenish pus escaped. The pus being thoroughly
washed out, both cysts were removed. The removal of the lower cyst was very difficult. After some separation it was possible to drag it up was very diffeult. After some separation it was possible to drag it up
from the deeper pirt of the nelvis. It was then found th he attached hv 1 Read befo:e the Medical So iety of Londo:. 